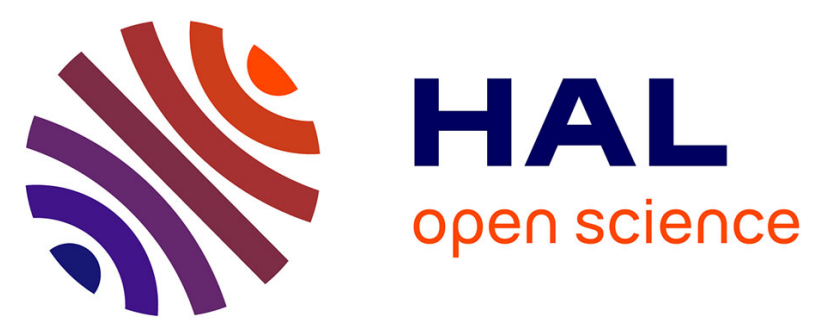

\title{
Empirical Mode Decomposition of Multiple ECG Leads for Catheter Ablation Long-Term Outcome Prediction in Persistent Atrial Fibrillation
}

Antonio Hidalgo-Muñoz, Ana Maria Tome, Decebal Gabriel Latcu, Vicente Zarzoso

\section{To cite this version:}

Antonio Hidalgo-Muñoz, Ana Maria Tome, Decebal Gabriel Latcu, Vicente Zarzoso. Empirical Mode Decomposition of Multiple ECG Leads for Catheter Ablation Long-Term Outcome Prediction in Persistent Atrial Fibrillation. 37th Annual International Conference of the IEEE Engineering in Medicine and Biology Society (EMBC-2015) , Aug 2015, Milan, Italy. pp.105-108, 10.1109/EMBC.2015.7318311 . hal-01302663

\section{HAL Id: hal-01302663 https://hal.science/hal-01302663}

Submitted on 14 Apr 2016

HAL is a multi-disciplinary open access archive for the deposit and dissemination of scientific research documents, whether they are published or not. The documents may come from teaching and research institutions in France or abroad, or from public or private research centers.
L'archive ouverte pluridisciplinaire HAL, est destinée au dépôt et à la diffusion de documents scientifiques de niveau recherche, publiés ou non, émanant des établissements d'enseignement et de recherche français ou étrangers, des laboratoires publics ou privés. 


\title{
Empirical Mode Decomposition of Multiple ECG Leads for Catheter Ablation Long-Term Outcome Prediction in Persistent Atrial Fibrillation
}

\author{
Antonio R. Hidalgo-Muñoz ${ }^{1}$, Ana M. Tomé ${ }^{2}$, Decebal G. Latcu ${ }^{3}$ and Vicente Zarzoso ${ }^{1}$
}

\begin{abstract}
Predictive models arouse increasing interest in clinical practice, not only to improve successful intervention rates but also to extract information of diverse physiological disorders. This is the case of persistent atrial fibrillation (AF), the most common cardiac arrhythmia in adults. Currently, catheter ablation (CA) is one of the preferred therapies to face this disease. However, selecting the best responders to $\mathrm{CA}$ by standard noninvasive techniques such as the electrocardiogram (ECG) remains a challenge. This work presents different predictive models for determining long-term CA outcome based on the dominant frequency (DF) of atrial activity measured in the ECG. The ensemble empirical mode decomposition (EEMD) is employed to obtain the intrinsic mode functions (IMFs) composing the ECG signal in each lead. The IMF DFs computed in multiple leads are then combined into a logistic regression (LR) model. The IMF DF features are discriminant enough to reach $\mathbf{7 9 \%}$ accuracy for long-term $\mathrm{CA}$ outcome prediction, outperforming other methods based on DF computation. Our study shows EEMD as a valuable alternative to extract clinically relevant spectral information from AF ECGs and confirms the advantage of $L R$ to build multivariate predictive models as compared with univariate analysis.
\end{abstract}

\section{INTRODUCTION}

Atrial fibrillation (AF) is the most common sustained arrhythmia encountered in clinical practice [1]. In order to achieve tailored protocols to treat AF, predicting their longterm outcome noninvasively is desirable. To this aim, electrocardiogram (ECG) analysis can provide valuable information.

Catheter ablation (CA) is one of the most effective techniques to face persistent AF. However, the criteria to select patients suffering from this AF form are not completely established in current guidelines [2]. Therefore, additional objective tools to aid in clinical decision making are necessary. Following this line, the CA outcome prediction problem in $\mathrm{AF}$ has been tackled from different standpoints. Some research works are mainly interested in finding out whether CA succeeds in terminating AF just after finishing the intervention [3, 4], whereas several predictive models have been rather aimed at determining long-term CA outcome $[5,6]$. This last issue has

\footnotetext{
${ }^{1}$ A. R. Hidalgo-Muñoz and V. Zarzoso are with the I3S Laboratory, University of Nice Sophia Antipolis (UNS), France \{hidalgo, zarzoso\} eiss.unice.fr

${ }^{2}$ A. M. Tomé is with the DETI/IEETA, University of Aveiro, Portugal ana@ua.pt

3 D. G. Latcu is with the Cardiology Department, Princess Grace Hospital, Monaco dg. latcuechpg.mc

A. R. Hidalgo-Muñoz is funded by a postdoctoral fellowship from the UNS. V. Zarzoso is a member of the Institut Universitaire de France.
}

considerable clinical relevance since it is linked to the patients' future wellness.

A number of features of the fibrillatory activity signal have been proposed to characterize AF, such as the f-wave mean amplitude $[6,7]$ among others. However, the AF ECG spectral domain is arguably one of the most studied aspects [8], as the AF cycle length has been directly linked to the atrial myocites' refractory period [9]. In general, AF spectral measures, and the AF dominant frequency (DF) in particular, have been usually computed manually [10], on ECG signals after QRST complex cancellation [11], or on an atrial activity source signal after applying blind source separation (BSS) techniques $[12,13]$.

In this work, we employ the ensemble empirical mode decomposition (EEMD) $[14,15]$ to obtain the intrinsic mode functions (IMFs) that compose the AF ECG signal without requiring any additional transformed space. The IMF DFs are used as the features included into the predictive model. In the ECG framework, EEMD has already been applied for ECG identification purposes [16]. A similar approach has been tested together with the computation of other statistical features to predict paroxysmal AF termination [17]. Nevertheless, only a single lead is considered in that study.

In the present approach we exploit the spatial variability of the automatically extracted EEMD features from multiple-lead ECG recordings. Specifically, the logistic regression (LR) technique [18] is used to design a binary classifier for discriminating the best responders to $\mathrm{CA}$ by combining spectral features from selected ECG leads. Results on an AF database show that this approach improves on univariate predictive models based on the DF feature.

\section{METHODS}

\section{A. ECG Recording, Ablation Procedure and Follow-up}

Sixty-two patients (52 male, $61.48 \pm 10.4$ years) having persistent and long-lasting persistent AF [1] treated at Princess Grace Hospital, Monaco, were included in the study and gave informed consent. The study was approved by the Institutional Committee on Human Research. One minute 12-lead ECG was acquired at a sampling rate of $977 \mathrm{~Hz}$ at the beginning of the CA procedure. ECG signals were filtered by a 4th-order zero phase bandpass Chebyshev filter with a lower cutoff frequency of $0.5 \mathrm{~Hz}$ and an upper cutoff of $40 \mathrm{~Hz}$ to remove baseline wander, power line, myoelectric interference and high frequency artifacts.

The ablation was performed with the aid of Prucka Cardiolab and Biosense CARTO electrophysiology 
measurement systems and was carried out in a stepwise manner [2], starting with circumferential pulmonary vein (PV) isolation with LASSO-guided disconnection, followed by fragmented potentials, non-PV triggers, roof line and mitral isthmus line right atrial ablation. AF termination during ablation was defined as sinus rhythm (SR) resumption or its change to a stable atrial tachyarrhythmia (AT). In cases without AF or AT termination, after catheter withdrawal a loading dose of amiodarone $(30 \mathrm{mg} / \mathrm{kg})$ was administered unless contraindicated. Direct current cardioversion was performed (150 to $200 \mathrm{~J}$, repeated up to 3 times under general anesthesia) if the arrhythmia was ongoing 24-48 $\mathrm{h}$ after CA.

All the patients underwent $\mathrm{CA}$ and were followed for $\mathrm{AF}$ recurrences during at least 6 months after ablation (follow up: $13.9 \pm 8.3$ months) in a "real-life" setting with repeated ECG and 24-h Holter monitoring to detect both clinical and asymptomatic recurrences. Long-term outcome is considered as CA success (positive group) when no AF recurrences are reported in the follow up of over 6 months, regardless of the procedural variability.

\section{B. Ensemble Empirical Mode Decomposition}

EEMD is a noise-assisted data analysis method consisting in adding white noise of finite amplitude to the ECG signals and then applying the empirical mode decomposition algorithm $[14,15]$. This procedure is repeated iteratively to reduce the influence of spurious artifacts. The added noise is treated as random noise. In this way, the $n$-th noisy observation, $n=\{1, \ldots, E\}$, is decomposed as

$$
x_{n}(t)=x(t)+\epsilon_{n}(t)=\sum_{j=1}^{J} c_{n}^{(j)}(t)+r_{n}(t)
$$

where $x(t)$ is the original signal (in our case, the AF ECG signal at a given lead), $\epsilon_{n}(t)$ is the random noise, $c_{n}^{(j)}$ represents the $j$-th IMF and $r_{n}(t)$ the residual trend obtained for the $n$-th noisy observation. Thus, the resultant IMF $c^{(j)}$ is computed by averaging all $c_{n}^{(j)}$ from the ensemble of $E$ noise iterations.

Therefore, EEMD requires setting two parameters, namely the size $E$ of the ensemble and a proper standard deviation $\sigma_{\text {noise }}=\gamma \cdot \sigma_{\text {signal }}$ of added Gaussian noise, where $\sigma_{\text {signal }}$ denotes the standard deviation of the signal amplitude distribution. Usually, both parameters are chosen empirically. This study used 10-s segment signals, $E=10$ and $\gamma=0.1$, typical values giving accurate outcomes [15]. On the other hand, a maximum of $J=8$ IMFs provided enough resolution for the typical AF frequency range of 3 to $9 \mathrm{~Hz}$. The IMF DF was determined as the maximal peak frequency of Welch's power spectral density estimate. EEMD was implemented in MATLAB 2014 (Mathworks, Natick, MA, USA).

\section{Logistic Regression}

LR is a probabilistic classification model aimed at predicting the outcome of a categorical dependent variable
(CA success or failure) based on one or more predictor variables. The next equation is an example for $M$ predictors:

$$
L R=\log \left(\frac{\theta}{1-\theta}\right)=b_{1} f_{1}+\cdots+b_{M} f_{M}
$$

where $\theta$ is the probability of belonging to the positive group (CA success), $b_{1}, \ldots, b_{M}$ are the coefficients of the regression model and $f_{1}, \ldots, f_{M}$ correspond to the DF values from the selected leads.

To build an adequate model, the number of variables should not be too large to avoid overfitting. In the framework of LR, the Wald index quantifies if the coefficient $b_{m}$ linked to the $m$-th variable can be considered statistically different from zero. Hence, we employ a backward elimination method that discards iteratively the least significant variable according to this index [18] until all the remaining variables are significant $(p<0.05)$.

\section{Statistical Analysis}

For the univariate analysis, distribution normality was checked by a Kolmogorov-Smirnov's test on both groups. Under data normality, groups were compared by a parametric Student's $t$-test, otherwise a Mann-Whitney $U$ test was used. Levene's correction was applied on $p$ values when homogeneity of variance could not be assumed. The area under the ROC curve (AUC) was used as a prediction performance index. This statistical analysis and the LR models were performed with SPSS version 13.0 (IBM, Armonk, NY, USA).

\section{RESULTS AND DISCUSSION}

\section{A. Intrinsic Mode Functions}

Fig. 1 shows the IMF DF values over the whole AF ECG database. The two first IMFs can be considered as high frequency noisy contributions and are not depicted in the figure. Of note, the averaged DFs of IMFs 5 and 6 belong to the range of interest for AF analysis (3 to $9 \mathrm{~Hz}$ ) [1]. Thus, only these two modes are taken into consideration next, since they present meaningful values for atrial activity characterization.

\section{B. Univariate Analysis}

In 15 out of the 62 patients AF recurrences were registered after $5.5 \pm 4.2$ months following CA $(75.8 \%$ of successful CA interventions). It is important to stress that long-term success includes patients who reached SR restoration or AT, as mentioned in Sec. II-A.

Table I shows the statistically significant differences between CA success and failure groups for EEMD DF values, as well as for DF values computed from single ECG leads after suppressing the QRST complex contribution with the adaptive singular value cancellation (ASVC) algorithm [11]. The rest of comparisons between single leads, including IMF 6, are not displayed as they present no statistically significant differences. The univariate analysis on the DF values computed from an atrial source signal extracted via RobustICA [12], a method based on BSS, and from an intracardiac electrogram (EGM) recorded in the left 


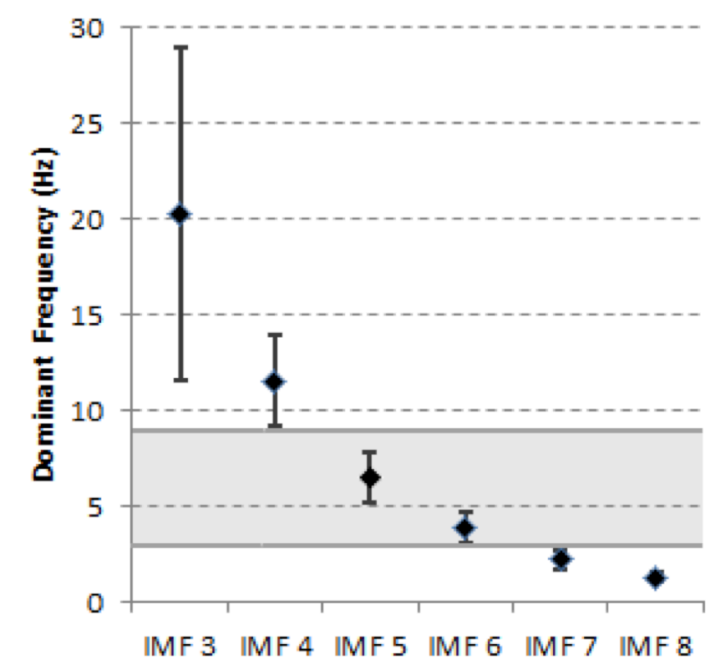

Figure 1. DF mean values ( $\pm \mathrm{SD}$ ) over the complete AF patient database from different IMFs computed in V1 $(E=10 ; \gamma=0.1)$. The horizontal solid lines mark the typical AF DF interval (3 to $9 \mathrm{~Hz}$ ).

atrial appendage, which could be considered as ground truth, are also included in Table I, together with the statistical contrast results. EGM DF values were computed following the method explained in [19]. Note that in the last two cases no significant differences exist, while the best performance is obtained by EEMD with IMF 5 in V2 yielding $\mathrm{AUC}=0.752$.

Unexpectedly, higher DF values are linked to long-term CA success in IMF 5 of lead V2 and in lead V1 after ASVC, which seems to contradict previous findings indicating that higher DFs tend to be related to AF recurrences [10]. This result suggests that the spatial variability of the atrial signal over ECG leads is an important factor to take into account in order to improve the reliability of spectral measures in the context of CA long-term outcome prediction. Indeed, such spatial variability has been acknowledged when dealing with f-wave amplitude features [6] and can actually be exploited to improve prediction performance [7]. The multivariate analysis of spectral features presented next points in the same direction.

\section{Multiple ECG Lead Predictive Models}

Table II details the performance and the selected leads by applying the LR approach with backward elimination (Section II.C) to the DF values obtained by EEMD in the suitable IMFs (5 and 6) and computed after ASVC. Note that the three LR models match up in the initial set size of $M=12$
TABLE II. LEADS RETAINED BY THE LR MODELS AND ASSOCIATED ACCURACY INDICES

\begin{tabular}{|l|c|c|c|}
\hline \multirow{2}{*}{ Leads } & \multicolumn{2}{|c|}{ EEMD } & \multirow{2}{*}{ ASVC } \\
\cline { 2 - 3 } & IMF 5 & IMF 6 & \\
\hline Area Under Curve (AUC) & aVL, V1, V2 & aVR, V1 & \multirow{2}{*}{ V1, V4 } \\
\hline Significance (p) & $\mathbf{0 . 8 4 5}$ & 0.700 & 0.801 \\
\hline Optimal Cutoff & $<0.001$ & 0.020 & $<0.001$ \\
\hline Positive Predictive Value & 0.72 & 0.75 & 0.72 \\
\hline Negative Predictive Value & $\mathbf{9 2 . 5} \%$ & $87.2 \%$ & $88.8 \%$ \\
\hline Sensitivity & $54.5 \%$ & $43.5 \%$ & $58.8 \%$ \\
\hline Specificity & $78.7 \%$ & $72.3 \%$ & $85.1 \%$ \\
\hline Accuracy & $80 \%$ & $66.7 \%$ & $66.7 \%$ \\
\hline
\end{tabular}

variables, corresponding to the DF value of every lead. Fig. 2 shows the ROC curves (with arrows pointing at the optimal cutoff points) of the mentioned LR models.

According to Table II, the simultaneous combination of DF values from several leads improves classification performance in every case. The best results are reached by combining DF values from IMF 5, being comparable to the prediction accuracy of other works reported in the literature $[6,7,10]$.

Regarding the most relevant leads for our classification task, one can remark the importance of the precordial leads, anatomically closest to the atria, and especially the selection of lead V1, one of the most used for AF analysis [20]. Either way, whereas the DF values of IMF 5 are closer to the expected fibrillatory frequency observed in the EGM recording, IMF 6 seems to be rather linked to a lower frequency modulation of the f-waves, whose physiological meaning remains to be clarified.

Note that the positive predictive values around $90 \%$ are higher than the initial proportion of the positives cases into the complete set of patients $(75.8 \%)$. Hence, a patient selection protocol based on these predictive models would consequently increase the rate of clinically successful CA procedures.

\section{CONCLUSIONS}

EEMD is presented as an alternative to BSS and ASVC techniques to extract spectral information from AF ECGs for predicting CA long-term outcome. In addition, the combination of DF values from either IMFs 5 or 6 by means of an LR model significantly improves the classification

TABLE I. STATISTICALLY SIGNIFICANT DIFFERENCES FOUND IN DOMINANT FREQUENCY VALUES BETWEEN GROUPS (UNIVARIATE ANALYSIS)

\begin{tabular}{|c|c|c|c|c|c|c|c|}
\hline & \multirow{2}{*}{ Lead } & \multicolumn{2}{|c|}{ Mean $(\mathrm{Hz}) \pm$ Standard Deviation } & \multirow{2}{*}{$\begin{array}{c}\text { Normal } \\
\text { distribution }\end{array}$} & \multirow{2}{*}{$p$-value } & \multicolumn{2}{|c|}{ ROC curve } \\
\hline & & CA Success & CA Failure & & & AUC & Significance $(p)$ \\
\hline EEMD (IMF 5) & V2 & $7.33 \pm 1.23$ & $5.97 \pm 1.54$ & Yes & 0.001 & 0.752 & 0.003 \\
\hline \multirow{2}{*}{ ASVC } & V1 & $5.79 \pm 0.88$ & $5.19 \pm 0.97$ & No & 0.029 & 0.662 & 0.060 \\
\hline & V4 & $5.34 \pm 1.38$ & $6.73 \pm 3.44$ & No & 0.023 & 0.692 & 0.026 \\
\hline RobustICA & $\mathrm{n} / \mathrm{a}$ & $5.44 \pm 1.27$ & $5.35 \pm 1.57$ & No & 0.510 & 0.559 & 0.548 \\
\hline Intracardiac & $\mathrm{n} / \mathrm{a}$ & $6.07 \pm 0.89$ & $6.30 \pm 1.01$ & No & 0.388 & 0.575 & 0.388 \\
\hline
\end{tabular}



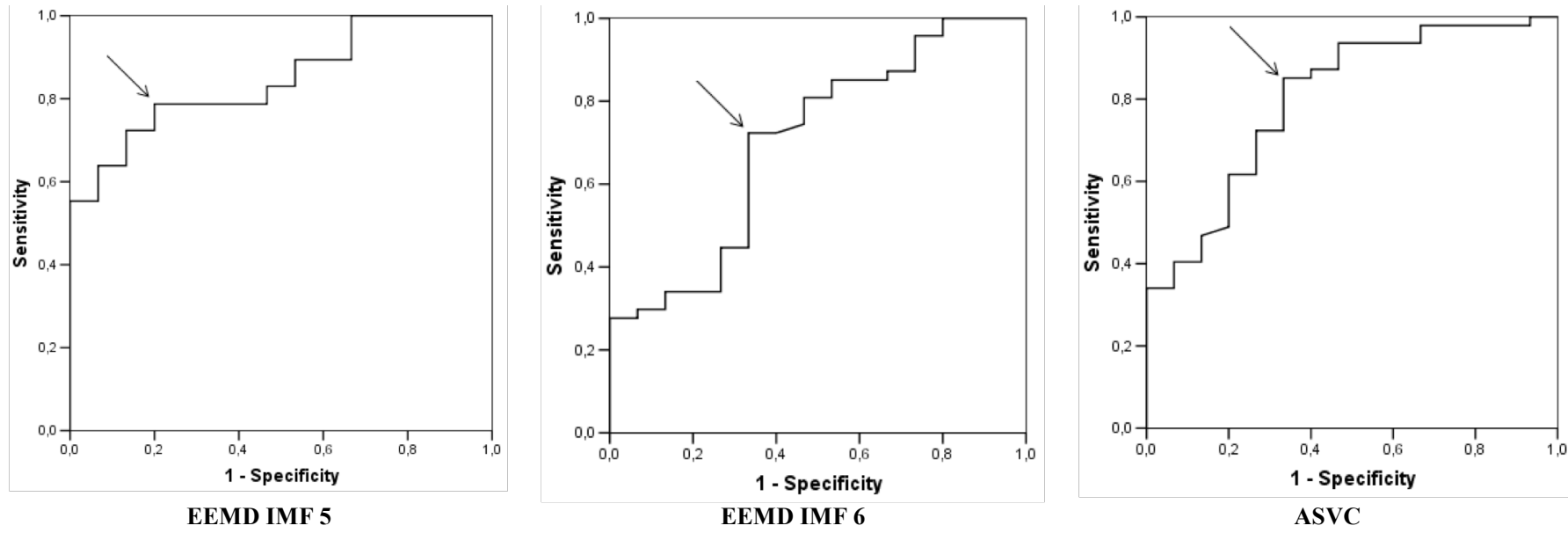

Figure 2. ROC curves from the corresponding LR score in the three multivariate models detailed in Table II.

accuracy. However, a prospective analysis would be necessary to confirm the generalization capability of this approach. Since the automatic feature extraction based on EEMD is cost effective, further works could explore other machine learning strategies by taking different features from the IMFs, such as spatiotemporal variability or spectral concentration indices.

\section{REFERENCES}

[1] C. T. January, et al, "2014 AHA/ACC/HRS guideline for the management of patients with atrial fibrillation: executive summary," J Am Coll Cardiol, vol. 64, no. 2 , pp. 2246-2280, 2014.

[2] H. Calkins, et al, "HRS/EHRA/ECAS Expert consensus statement on catheter and surgical ablation of atrial fibrillation: recommendations for patient selection, procedural techniques, patient management and follow-up, definitions, endpoints, and research trial design," Europace, vol.14, pp. 528-606, 2012.

[3] A. Buttu, et al., "Termination of atrial fibrillation by catheter ablation can be successfully predicted from baseline ECG," in Proc. Comp Cardiol, Krakow, Poland, 2012, 39, pp.189-192.

[4] E. K. Heist, F. Chalhoub, C. Barrett, S. Danik, J. N. Ruskin, and M. Mansour, "Predictors of atrial fibrillation termination and clinical success of catheter ablation of persistent atrial fibrillation," Am J Cardiol vol. 110, no. 4, pp. 545-551, 2012.

[5] A. N. Ganesan, et al., "Long-term outcomes of catheter ablation of atrial fibrillation: a systematic review and metaanalysis," J Am Heart Assoc vol. 2, no. 2, e004549, 2013.

[6] I. Nault, et al, "Clinical value of fibrillatory wave amplitude on surface ECG in patients with persistent atrial fibrillation," $J$ Interven Card Electrophysiol, vol. 26, no. 1, pp. 11-19, 2009.

[7] M. Meo, V. Zarzoso, O. Meste, D. G. Lactu, and N. Saoudi, "Spatial variability of the 12-lead surface ECG as a tool for noninvasive prediction of catheter ablation outcome in persistent atrial fibrillation," IEEE Trans Biomed Eng vol. 60, pp. 20-27, 2013.

[8] L. Y. Di Marco, D. Raine, J. P. Bourke, and P. Langley, "Characteristics of atrial fibrillation cycle length predict restoration of sinus rhythm by catheter ablation," Heart Rhythm vol. 10, no. 9, pp. 1303-1310, 2013.
[9] A. Bollmann, N. K. Kanuru, K. K. McTeague, P. F. Walter, D. B. DeLurgio and J. J. Langberg, "Frequency analysis of human atrial fibrillation using the surface electrocardiogram and its response to ibutilide," Am J Cardiol, vol. 8, pp. 14391445, 1998.

[10] S. Matsuo, et al, "Clinical predictors of termination and clinical outcome of catheter ablation for persistent atrial fibrillation," J Am Coll Cardiol vol. 54, pp. 788-795, 2009.

[11] R. Alcaraz, and J. J. Rieta, "Adaptive singular value cancelation of ventricular activity in single-lead atrial fibrillation electrocardiogram," Physiol Meas, vol. 29, no. 12, pp. 1351-1369, 2008.

[12] V. Zarzoso, and P. Comon, "Robust independent component analysis by iterative maximization of the kurtosis contrast with algebraic optimal step size," IEEE Trans Neural Netw, vol. 21, no. 2, pp. 248-261, 2010.

[13] M. Garibaldi, V. Zarzoso, D. G. Latcu, and N. Saoudi, "Predicting catheter ablation outcome in persistent atrial fibrillation using atrial dominant frequency and related spectral features," in Proc. Annu Int Conf IEEE Eng Med Biol Society (EMBC), San Diego, CA, USA, 2012, pp. 613-616.

[14] N. E. Huang, et al., "The empirical mode decomposition and the Hilbert spectrum for nonlinear and non-stationary time series analysis," in Proc Royal Soc London. Ser A: Math, Phys Eng Sci, 1998, vol. 454, no. 1971, pp. 903-995.

[15] Z. Wu, and N. E. Huang, "Ensemble empirical mode decomposition: a noise-assisted data analysis method," $A d v$ Adaptive Data Anal, vol. 1, no. 1, pp.1-41, 2009.

[16] Z. Zhao, L. Yang, D. Chen, and Y. Luo, "A human ECG identification system based on ensemble empirical mode decomposition," Sensors, vol. 13, no. 5, pp. 6832-6864, 2013.

[17] M. Mohebbi, and H. Ghassemian, "Predicting termination of paroxysmal atrial fibrillation using empirical mode decomposition of the atrial activity and statistical features of the heart rate variability," Med Biol Eng Comput, vol. 52, pp. 415-427, 2014.

[18] A. Indrayan. Medical Biostatistics. CRC Press, 3rd Ed., 2012.

[19] G. W. Botteron, and J. M. Smith, "A technique for measurement of the extent of spatial organization of atrial activation during atrial fibrillation in the intact human heart," IEEE Trans Biomed Eng, vol. 42, no. 6, pp. 579-586, 1995.

[20] S. Petrutiu, J. Ng, G. M. Nijm, H. Al-Angari, S. Swiryn, and A.V. Sahakian. "Atrial fibrillation and waveform characterization," IEEE Eng Med Biol Magazine, vol. 25, no. 6, pp. 24-30, 2006. 